Research Articles

\title{
Hemodynamic Catheters: The Reprocessing, Cleanliness and in vitro Biofilm Formation by Enterococcus faecium in a Continuous Flow Model
}

\author{
${ }^{1}$ Cleber Galdino da Silva, ${ }^{1}$ Edson Pires de Oliveira, ${ }^{1}$ Ednalva Miranda Guizi ${ }^{2}$ Julio Spadotto \\ ${ }^{2}$ Celia Guadalupe Tardeli de Jesus Andrade, ${ }^{3}$ Gilselena Kerbauy, ${ }^{3}$ Vivian Biazon EI Reda Feijó \\ ${ }^{3}$ Elizabeth Silva Ursi, ${ }^{3}$ Renata Aparecida Belei, ${ }^{4}$ Glenda Cavalari Simões \\ ${ }^{4}$ Alane Tatiana Pereira Moralez, ${ }^{4}$ Galdino Andrade and ${ }^{4}$ Admilton Gonçalves de Oliveira \\ ${ }^{1}$ Instituto de Ensino Superior de Londrina, Londrina, Brazil \\ ${ }^{2}$ Laboratory for Microscopy and Microanalysis, Universidade Estadual de Londrina, Londrina, Brazil \\ ${ }^{3}$ Universidade Estadual de Londrina, University Hospital and Clinic Center, Londrina, Brazil \\ ${ }^{4}$ Department of Microbiology, Universidade Estadual de Londrina, Londrina, Brazil
}

Article history

Received: 25-12-2014

Revised: $12-01-2015$

Accepted: 08-07-2015

Corresponding Author: Admilton Gonçalves de Oliveira, Department of Microbiology, Universidade Estadual de Londrina, Londrina, Brazil

Email: admilton@uel.br

\begin{abstract}
Reprocessing single-use devices to cut costs is a common practice in hospitals around the world. In Brazil, there are few studies of reprocessing hemodynamic catheters and thus, this study aimed to evaluate the effectiveness of reprocessing hemodynamic catheters before and after biofilm formation in vitro using a continuous flow model. We used a sterility test and Scanning Electron Microscopy (SEM) to assess the presence of microorganisms, residue and integrity of a New (NC) and Reprocessed (RC) hemodynamic catheter, before and after in vitro biofilm formation by a clinical isolate of Enterococcus faecium (strain 155). NC was considered the negative control. The sterility test did not show the presence of microorganisms in either catheters used as a negative control (NC and RC). On the other hand, changes in integrity were observed by $\mathrm{SEM}$ in the $\mathrm{RC}$, with a large number of microcracks and recesses, indicating that this would get worse after reprocessing. After biofilm formation and subsequent sterilization by ethylene oxide, both catheters were examined by SEM and RC showed a dense array of exopolysaccharide and substantial organic waste material, which was not evident in NC, showing changes in surface integrity. Ethylene oxide sterilization is very efficient in the sterilization process but the reprocessed catheters after biofilm formation by strain 155, showed marked surface changes, which increases the adhesion of organic matter and compromises the cleaning process in reprocessing. The results can be used as a parameter for hospitals and companies that reprocess catheters, to develop protocols for standardized and systematic surveillance in reusing materials recommended for single use to prevent infections.
\end{abstract}

Keywords: Single-use Devices, Catheter-Associated Infections, Sterilization, Residue, Scanning Electron Microscopy

\section{Introduction}

Reusing or not a manufactured product for medical and hospital has been a global question. There are those who support this practice aimed at reducing costs, since these products are quite costly for hospitals. However, there are many who question the lack of safety in the reprocessing of these products, where the cleaning and sterilization methods are not always effective in eliminating the presence of contaminants, which may cause serious problems for the patient, where a reprocessed and reused product is involved, such as the bacterial contamination established in biofilms and toxic waste.
(C) 2015 Cleber Galdino da Silva, Edson Pires de Oliveira, Ednalva Miranda Guizi, Julio Spadotto, Celia Guadalupe Tardeli de Jesus Andrade, Gilselena Kerbauy, Vivian Biazon El Reda Feijó, Elizabeth Silva Ursi, Renata Aparecida Belei, Glenda Cavalari Simões, Alane Tatiana Pereira Moralez, Galdino Andrade and Admilton Gonçalves de Oliveira. This open access article is distributed under a Creative Commons Attribution (CC-BY) 3.0 license. 
Catheter-associated infections cause considerable morbidity and mortality in hospitalized patients. One of the difficulties associated with catheter infection is to establish the diagnosis. Evidence that a catheter is associated with an infection can be obtained when the catheter is removed and the distal end is subjected to microbiological evaluation. The isolation of the same microorganism from the tip of the catheter and the blood indicates a catheter-related infection (Elliott et al., 2000). In this regard, many methods are available for the culture of the catheter tip (Cleri et al., 1980; Collignon el al., 1986; Maki et al., 1977; Raad et al., 1992). However, the gold standard is the culture of the catheter tip described by (Schinabeck and Ghannoum, 2003).

Bacteremia has been increasingly associated with the use of catheters from the continuous use of the gold standard for catheter tip culture. In this context, infection by vancomycin-resistant Enterococcus (VRE) arises as a significant problem among hospitalized patients (Edmond et al., 1996; Fridkin et al., 1999; Montecalvo et al., 1994; Newell et al., 1998). Furthermore, the rate of enterococcal bacteremia associated with central venous catheters increased progressively during the past two decades, (Gray et al., 1994; Malone et al., 1986; Patterson et al., 1995; Shlaes et al., 1981).

Thus, the use of Enterococcus faecium in vitro biofilm experiments is of high importance, since bacteremia caused by E. faecium associated with catheters has caused great concern in recent years, not only because of the difficulty of controlling the microorganism but also because of its virulence.

Gray et al. (1994) reported in their study that 35\% of catheter-related infections of the bloodstream were caused by enterococci. Moreover, several studies have reported VRE episodes related to catheter use (Edmond et al., 1996; Lai, 1996; Moellering et al., 1999; Sandoe et al., 2002).

The most important factor in the pathogenesis of infections associated with foreign objects is the ability of bacteria to form biofilms on different surfaces of such devices. Biofilm is defined as a layered community of sessile microorganisms in an extracellular polymeric matrix produced by the microorganism, formed in an organized process (Donlan and Costerton, 2002). This process starts with the rapid and primary adhesion of bacterial cells onto the substrate surface followed by extracellular matrix coating. Next, the bacteria proliferate and accumulate, forming multilayered cell aggregates incorporated into the extracellular matrix (Speziale et al., 2008).

Studies have focused on standardization and researchers working in the area of reusing single-use materials have published the validation of cleaning products for repeated reuse. The cleaning process, whether manual or automated, has as main objective the elimination of possible waste and/or protein aggregates that could generate sources of contamination, or even facilitate the formation of new biofilm in the lumen of the reprocessed catheter (Alfa and Nemes, 2003).

Simulating a process is one of the most effective ways to conduct an evaluation, as well as finding the best way to achieve low levels of contamination that do not cause reactions or infection in the patient. Accordingly, this study aimed to evaluate possible changes on the inner surface of reprocessed and sterilized hemodynamic catheters and formation of biofilm by the clinical isolate E. faecium $155 \mathrm{VRE}$ in an in vitro continuous flow model before and after reprocessing and sterilization using ethylene oxide.

\section{Material and Methods}

\section{Bacterial Strain}

The bacterial strain of biofilm formation used in the experiments was the clinical isolate E. faecium $155 \mathrm{VRE}$ originating from cultures that were discarded as biological waste at the Laboratory of Microbiology of University Hospital and Clinic Center, Londrina, Brazil. These wastes were from tests performed in the routine care of hospitalized patients. Samples did not have any information that could identify the patient. The clinical isolate was kept in $40 \%$ glycerol at $-4^{\circ} \mathrm{C}$.

\section{Culture Medium and Inoculum}

The culture medium used in the experiments with in vitro of continuous flow and microbiological testing was Brain Heart Infusion (BHI). The inoculum of E. faecium, 155 , was prepared from a liquid culture of cells in exponential growth phase. The initial inoculum for the continuous flow was adjusted to $10^{4}$ (colony forming units) $\mathrm{CFU} / \mathrm{mL}$ in a spectrophotometer at $600 \mathrm{~nm}$.

\section{Characteristics of the Catheter}

The present study was carried using hemodynamic catheters (Performa ${ }^{\circledR}$ 5f, VERT). The unused catheters were obtained straight from the supplier and those reused five times were kindly donated to us by University Hospital and Clinic Center, Londrina, Brazil.

The new catheters and reprocessed catheters were evaluated by Scanning Electron Microscopy (SEM) in two steps, using three random replicas each. In the first (controls), we evaluated the New Catheter (NC) and the reprocessed catheter reused five times (RC). In the second (assay), samples of the NC and RC were subjected to biofilm formation by continuous flow, nominees were NCB and RCB. Afterwards, subsamples were subjected to direct evaluation by SEM and other subsamples were subjected to reprocessing (NCBR and RCBR). Subsamples of the NCBR and RCBR were subjected to sterility assay and other subsamples were evaluated by SEM (Fig. 1). 


\section{In Vitro Continuous Flow Model}

For biofilm formation, a continuous flow system was constructed by joining the catheters to an Erlenmeyer flask (feed flask) containing BHI liquid medium with inoculum of $E$. faecium, strain 155 VRE, adjusted to $10^{4} \mathrm{CFU} / \mathrm{mL}$ and another Erlenmeyer flask (receiver flask) (Fig. 2).

For in vitro biofilm formation, the flow was set at $1 \mathrm{~mL} / \mathrm{min}$ using a pressure pump. Afterwards, the inoculum was carried through each catheter for $15 \mathrm{~h}$ and the whole system was maintained at $37^{\circ} \mathrm{C}$. The

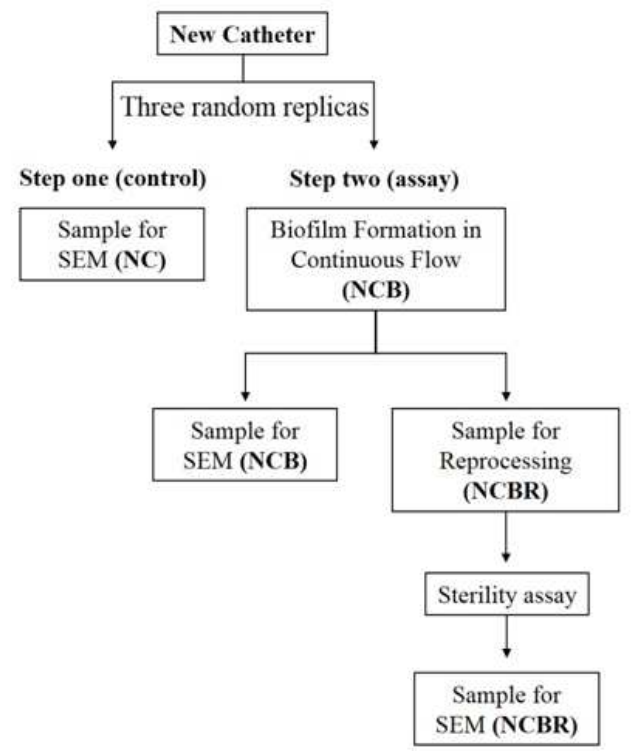

guidelines of Agência Nacional de Vigilância Sanitária - ANVISA (Brasil, 2012) were used for reprocessing and sterilization of catheters.

\section{Processing Protocol}

Following the continuous flow experiments, the samples for reprocessing were placed, for ten minutes, in a warmed container filled with $4 \mathrm{~mL}$ of enzymatic detergent (Max Zyme ${ }^{\circledR}$ ). Afterwards, the samples were dried and forwarded to a private company to be reprocessed and sterilized by ethylene oxide.

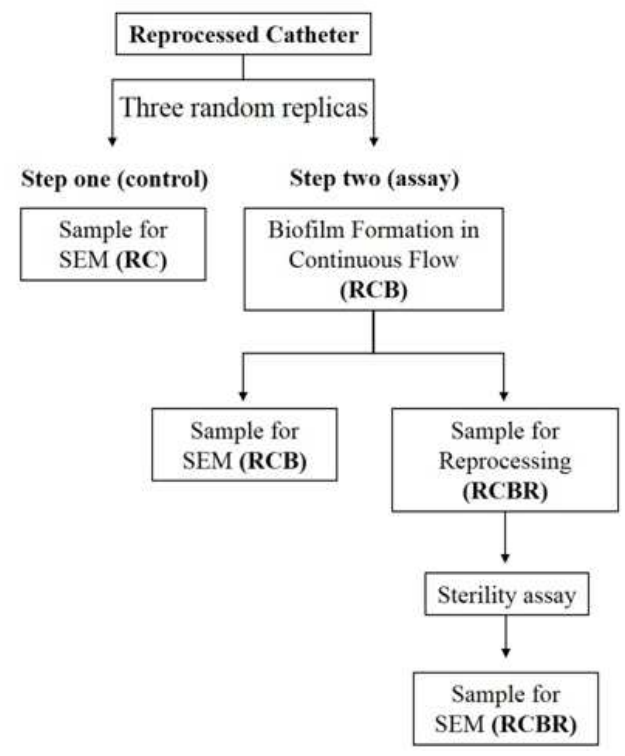

Fig. 1. Organizational chart of the assay

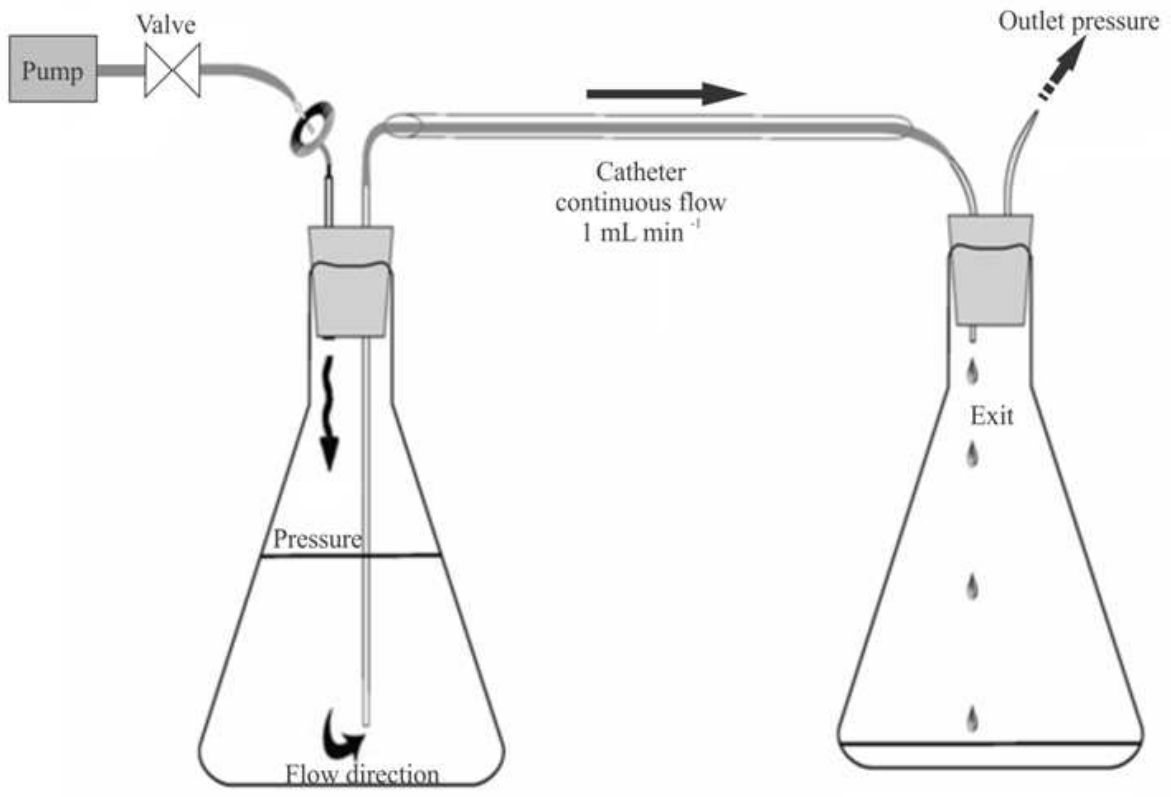

Fig. 2. Schematic depiction of the continuous flow model, including pressure pump, feeding and waste flasks and angioplasty catheter 


\section{Microbiological Evaluation}

Samples were aseptically divided into $5 \mathrm{~cm}$ pieces, where 3 random replicas were used for microbiological evaluation and further incubated in BHI liquid medium for $24 \mathrm{~h}$ at $37^{\circ} \mathrm{C}$. Next, microbial growth was determined according to medium turbidity.

\section{Scanning Electron Microscopy}

The samples for Scanning Electron Microscopy (SEM) evaluation were fixed by immersion in $2.5 \%$ glutaraldehyde in $0.1 \mathrm{M}$ phosphate buffer, $\mathrm{pH} 7$, for $12 \mathrm{~h}$ and post-fixed in $1 \% \mathrm{OsO}_{4}$ for one hour. The fixed material was then dehydrated in an ethanol gradient $(70,80,90$ and $100 \mathrm{GL})$. Next, the samples were critical point dried with $\mathrm{CO}_{2}$ (BALTEC CPD 030 Critical Point Dryer), fixed on a support, coated with gold (BALTEC SDC 050 Sputter Coater) and examined by SEM (FEI Quanta 200).

\section{Results}

The strain $155 \mathrm{VRE}$, formed a high biofilm amount on the surface of the angioplasty catheters tested. The sterility testing of NCBR and RCBR samples, which were subjected to the in vitro biofilm formation test and then reprocessed and sterilized by ethylene oxide, showed no microbial growth after incubation.

SEM demonstrated that the NC surface was free of residue (Fig. 3A). In the higher magnification was observed a intact surface (Fig. 3B). On the other hand, was found great microcracks, roughness and residue inside the lumen of RC (catheter reused five times) in the small and in the higher magnification (Fig. 3C and 3D).

After biofilm formation by E. faecium VRE 155 in a continuous flow model, SEM analysis revealed an exopolysaccharide matrix on NCB (Fig. 4A and 4B). However, a large amount of this material was observed in the RCB (Fig. 4C and 4D). This suggests that the numerous microcracks and roughness on the RCB foster the adherence and establishment of VRE 155.

After reprocessing, was observed that NCBR was almost completely free of residue (Fig. 5A). However, already had small alterations in the lumen (Fig. 5B). On the other hand, in the RCBR was observed high amount of residue, roughness, microcracks and a large deformation (Fig. 5C and 5D). These observed changes by electron microscopy indicate that repeated processing render useless of the catheter.
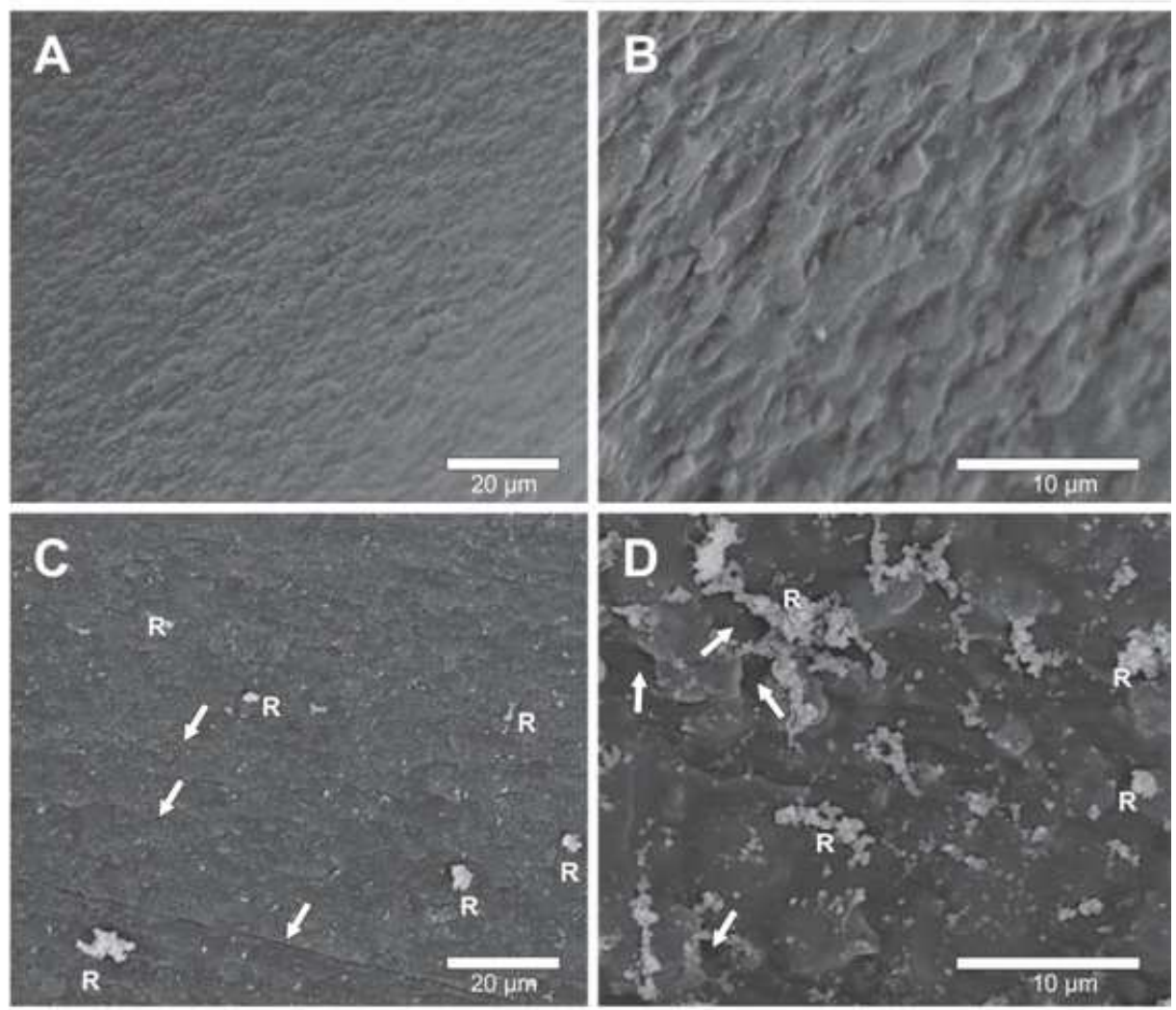

Fig. 3. Scanning electron microscopy of angioplasty catheter. A: new catheter (NC); B: higher magnification of NC; C: reprocessed catheter reused five times (RC); D: higher magnification RC. Arrow: microcracks; R: residue 

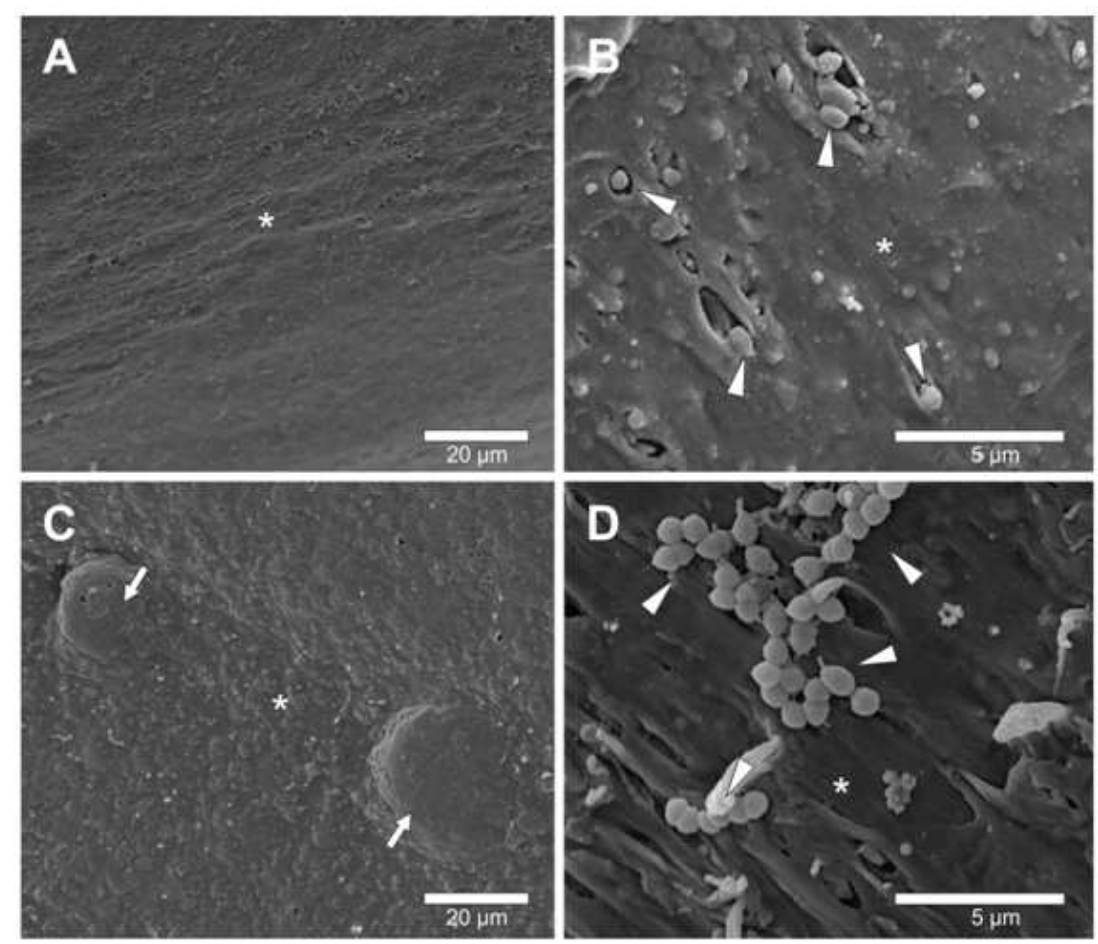

Fig. 4. Scanning electron microscopy of angioplasty catheter. A: new catheter subjected to biofilm formation by continuous flow (NCB); B: higher magnification of NCB; C: reprocessed catheter reused five times subjected to biofilm formation by continuous flow (RCB); D: higher magnification of RCB. Asterisk: biofilm layer; Arrow: mature biofilm; Arrowhead: E. faecium strain 155 VRE
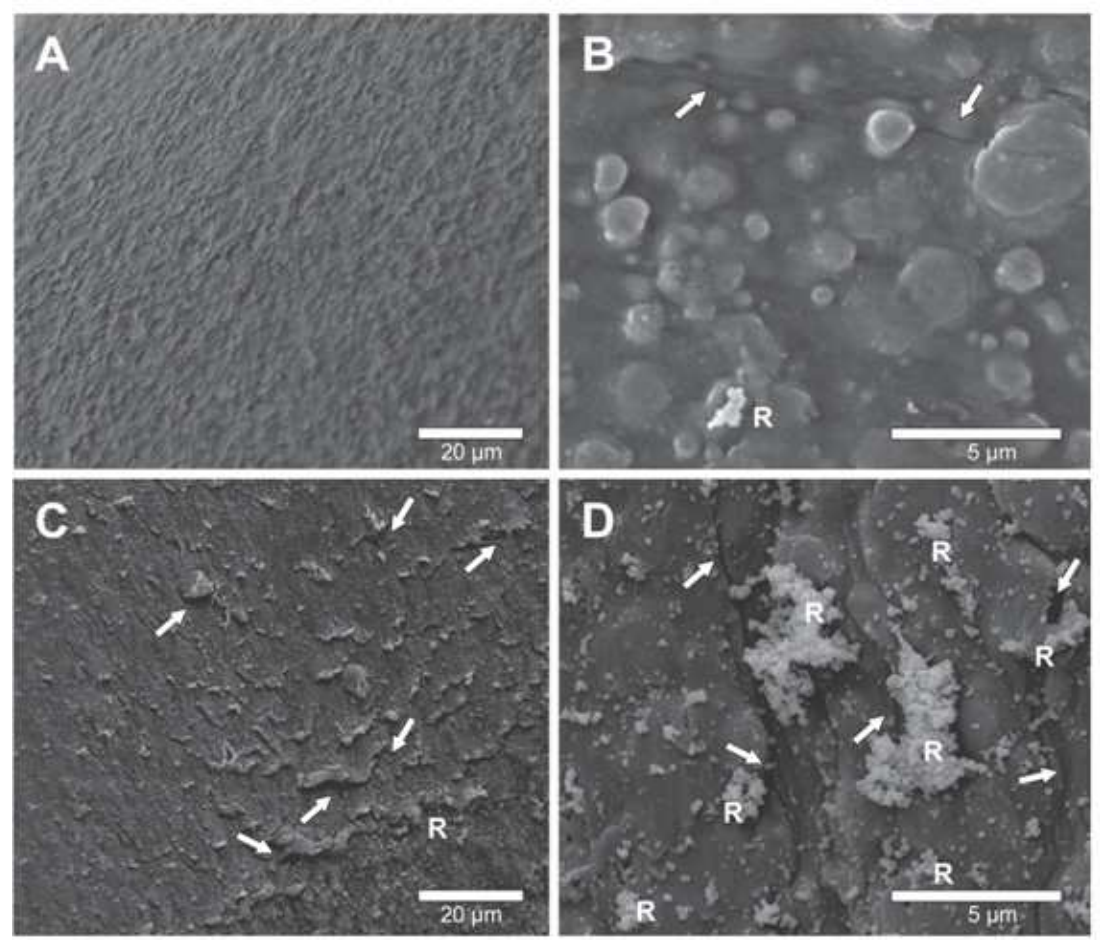

Fig. 5. Scanning electron microscopy of angioplasty catheter. A: new catheter subjected to biofilm formation by continuous flow and reprocessed (NCBR); B: higher magnification of NCBR; C: reprocessed catheter reused five times subjected to biofilm formation by continuous flow and reprocessed (RCBR); D: higher magnification of RCBR; Arrow: microcracks; R: residue 


\section{Discussion}

Catheterism is one of the best discoveries in recent decades for diagnosis and therapy fields. The clinical procedure and manufacturer stipulate that a catheter is for a single use. Catheters are expensive and to decrease costs, many hospitals choose to reuse catheters (Pinto and Graziano, 2000). The risk of reprocessing this kind of accessory could contribute to increased risk of infection of the patient even though the catheter is cleaned up and sterilized, guaranteeing the absence of microorganisms or toxic agents and, especially, the retention of the functional characteristics of such products for reuse (Silva, 2005).

The presence of residue on reused catheters permits the adherence of bacteria as shown in the present study. The residue could contain exogenous pyrogens produced by bacterial metabolism. The large an amount of residue found in reprocessed catheters (before and after the continuous flow in biofilm formation test) could explain the importance of single use of the accessory.

Many authors have reported the difficulty of cleaning devices, in assessing the problems on the surface of reused catheters (Krause et al., 2000; Penna and Ferraz, 2001). By microscopy, it was possible to observe the presence of many cracks and roughness, which promote the adhesion of organic matter, decreasing the efficiency of cleaning processes.

In this study, we found that the catheters after reprocessing showed the presence of substantial residue, which leads us to conclude that reprocessing procedures are relatively inefficient in cleaning the catheter lumen. However, a study comparing infection risk in patients undergoing cardiac catheterization with disposable versus reprocessed catheters found that no patient developed infection (Frank et al., 1988). Similarly, in a survey of 12 large medical centers, reuse of pacing catheters was common and was not associated with postprocedure infections (O'Donoghue and Platia, 1988).

According to Brazil's regulatory agency (Brasil, 2012), the processing of catheters demands a validation protocol granted by the Health Bureau. However, even though the reuse of medical devises is common in developing countries, the procedures do not assure catheter integrity, as demonstrated in this study.

For many devices that are commonly reused, clear protocols for reprocessing and sterilization exist. However, for many single-use devices, such protocols do not exist and institutions that reprocess such devices may not even have their own internal protocols.

According to Hussain et al. (2012) if a product can be economically reprocessed with validated protocols and deemed to be functional, there is no reason to discard it after one use. If working models of safe sterilization and reprocessing can be achieved, it will be of use to both the patient and the environment. On the other hand,
Shuman and Chenoweth (2012) believe that there may be cost savings, but that the degree to which savings are offset by adverse events is uncertain. More research is needed to help answer these important questions. In addition, there are many potential legal and ethical issues related to the reuse of single-use devices, again stemming from the lack of standards and data regarding adverse events.

According to Pantos et al. (2013), the reuse of Percutaneous Coronary Intervention (PCI) catheters is not generally recommended, since there are contradictory conclusions as far as patient safety is concerned.

Between December 1999 and July 2001, Amarante et al., (2008) conducted a survey using a questionnaire that was sent to 240 institutions affiliated with the Brazilian Society of Hemodynamics and Interventional Cardiology. Of the 119 institutions that participated in the survey, 97\% stated that they reprocessed single-use utensils. Of these, $20 \%$ reported reuse less than five times before disposal, $38 \%$ reuse ranging from 5 to 10 times, $15 \%$ reuse from 11 to 20 times and $11 \%$ over twenty-times reuse. In that same survey, $13 \%$ reported having no control over reuse.

The safety conditions presented by the reuse of single-use item still expose the patient to infection risk, as suggested by Greene (2004). The concerns of the Association for the Advancement of Medical Instrumentation (AAMI) with regard to reuse include infection, pyrogens, toxic residue, functional reliability and physical integrity.

\section{Conclusion}

The results of this study showed that reprocessed catheters have cracks and roughness, which foster the retention of organic material with potential risk to the patient. Considering these results, the reuse of catheters should be discouraged by health institutions, avoiding any threat to the patient's health. In addition, this study contributes to promoting awareness in hospitals about the concerns with reuse of catheters in clinical procedures, about establishing new protocols for reprocessing catheters and about systematic surveillance of single-use utensils. The present study allows the reader to reflect on the questionable efficiency of reprocessing and number of points that should prompt institutes to police the reuse of all single-use devices.

\section{Acknowledgment}

The authors are grateful to the University Hospital and Clinic Center, Londrina, Brazil and Sterilab Material Sterilization Medical LTD, Londrina, Brasil. The authors would also like to thank the Laboratory for Electron Microscopy and Microanalysis-LMEM UEL/FINEP for help with the SEM experiments. Dr. A. Leyva helped with English editing of the manuscript. 


\section{Funding Information}

We thank the National Council of Scientific, Technological and Inovation (CNPq).

\section{Author's Contributions}

CleberGaldino da Silva: Laboratory tests and manuscript preparation.

Edson Pires de Oliveira: Laboratory tests and manuscript preparation.

Ednalva Miranda Guizi: Laboratory tests.

Julio Spadotto: Electron microscopy analysis.

Celia Guadalupe Tardeli de Jesus Andrade: Coordinated the electron.

Gilselena Kerbauy: Angioplasty catheter assays.

Vivian Biazon El RedaFeijó: Angioplasty catheter assays.

Elizabeth Silva Ursi: Angioplasty catheter assays.

Renata Aparecida Belei: Angioplasty catheter assays.

Glenda Cavalari Simões: Laboratory tests.

Alane Tatiana Pereira Moralez: Coordinated the in vitro continuous.

Galdino Andrade: Data-analysis and manuscript revision.

AdmiltonGonçalves de Oliveira: Designed the research plan, organized.

\section{Ethics}

This article is original and contains unpublished material. The corresponding author confirms that all of the other authors have read and approved the manuscript and no ethical issues involved.

\section{References}

Alfa, M.J. and R. Nemes, 2003. Inadequacy of manual cleaning for reprocessing single-use, triple-lumen sphinctertomes: Simulated-use testing comparing manual with automated cleaning methods. Am. J. Infection Control 31: 193-207.

DOI: $10.1067 / \mathrm{mic} .2003 .22$

Amarante, J.M.B., C.M. Toscano, M.L. Pearson, V. Roth and W.R. Jarvis et al., 2008. Reprocessing and reuse of single-use medical devices used during hemodynamic procedures in Brazil: A widespread and largely overlooked problem. Infection Control Hospital Epidemiol., 29: 854-858. DOI: 10.1086/590357

Brasil, 2012. ANVISA-Agência Nacional de Vigilância Sanitária. Resolução $\mathrm{n}^{\circ} 15$, de 15 de março de 2012, Dispõe sobre requisitos de boas práticas para o processamento de produtos para saúde e dá outras providências.
Cleri, D.J., M.L. Corrado and S.J. Seligman, 1980. Quantitative culture of intravenous catheters and other intravascular inserts. J. Infect. Dis., 6: 781786. DOI: $10.1093 /$ infdis/141.6.781

Collignon, P.J., N. Soni, I.Y. Pearson, W.P. Woods and R. Munro et al., 1986. Is semiquantitative culture of central vein catheter tips useful in the diagnosis of catheter-associated bacteremia?. J. Clin. Microbiol., 24: 532-535.

Donlan, R.M. and J.W. Costerton, 2002. Biofilms: Survival mechanisms of clinically relevant microorganisms. Clin. Microbiol. Rev., 15: 167-193. DOI: $10.1128 / C M R .15 .2 .167-193.2002$

Edmond, M.B., J.F. Ober, J.D. Dawson, D.L. Weinbaum and R.P. Wenzel, 1996. Vancomycin-resistant enterococcal bacteremia: natural history and attributable mortality. Med. Clin. Infect. Dis., 23: 1234-1239. DOI: 10.1093/clinids/23.6.1234

Elliott, T.S.J., S.E. Tebbs, H.A. Moss, T. Worthington and M.K. Spare et al., 2000. A novel serological test for the diagnosis of central venous catheter-associated sepsis. J. Infect., 40: 262-266. DOI: 10.1053/jinf.2000.0656

Frank, U., L. Herz and F.D. Daschner, 1988. Infection risk of cardiac catheterization and arterial angiography with single and multiple use disposable catheters. Clin. Cardiol., 11: 785-787.

DOI: $10.1002 /$ clc.4960111111

Fridkin, S.K., C.D. Steward, J.R. Edwards, E.R. Pryor and J.E. McGowan et al., 1999. Surveillance of antimicrobial use and antimicrobial resistance in united states hospitals: Project ICARE phase 2. Clin. Infect. Dis., 29: 245-252. DOI: 10.1086/520193

Gray, J., P.J. Marsh, D. Stewart and S.J. Pedler, 1994. Enterococcal bacteraemia: A prospective study of 125 episodes. J. Hospital Infection 27: 179-186. DOI: 10.1016/0195-6701(94)90125-2

Greene, V.W., 2004. Reuse of devices labeled for singles-use. In: Mayhall CG Hospital Epidemiology and Infection Control. 3. Ed. Lippincott: Williams and Wilkins, 2004, 1535-1545.

Hussain, M., K.P. Balsara and S. Nagral, 2012. Reuse of single-use devices: Looking back, looking forward. National Med. J. India 25: 151-155.

Krause, G., G. Dziekan and F.D. Daschner, 2000. Reuse of coronary angioplasty balloon catheters: yes or no? European Heart J., 21: 185-189.

Lai, K.K., 1996. Treatment of vancomycin-resistant Enterococcus faecium infections. Archives Internal Med., 156: 2579-2584.

Maki, D.G., C.E. Weise and H.W. Sarafin, 1977. A semiquantitative culture method for identifying intravenous-catheter-related infection. New England J. Med., 296: 1305-1309.

DOI: 10.1056/NEJM197706092962301 
Malone, D.A., R.A. Wagner, J.P. Myers and C. Watanakunakorn, 1986. Enterococcal bacteremia in two large community teaching hospitals. Am. J. Med., 81: 601-606. DOI: 10.1016/0002-9343(86)90544-9

Moellering, R.C., P.K. Linden and J. Reinhardt, 1999. The efficacy and safety of quinupristin/dalfopristin for the treatment of infections caused by vancomycin-resistant Enterococcus faecium. J. Antimicrobial Chemotherapy 44: 251-261. DOI: $10.1093 / \mathrm{jac} / 44.2 .251$

Montecalvo, M.A., H. Horowitz, C. Gedris, C. Carbonaro and F.C. Tenover et al., 1994. Outbreak of vancomycin-, ampicillin- and aminoglycoside-resistant Enterococcus faecium bacteremia in an adult oncology unit. Antimicrobial Agents Chemotherapy, 38: 13631367. DOI: 10.1128/AAC.38.6.1363

Newell, K.A., J.M. Millis, P.M. Arnow, D.S. Bruce and E.S. Woodle et al., 1998. Incidence and outcome of infection by vancomycin-resistant Enterococcus following orthotopic liver transplantation. Transplantation 65: 439-442.

O'Donoghue, S. and E.V. Platia, 1988. Reuse of pacing catheters: a survey of safety and efficacy. Pacing Clin. Electrophysiol., 11: 1279-1280. DOI: $10.1111 / \mathrm{j} .1540-8159.1988 . t b 03988 . \mathrm{x}$

Pantos, I., E.P. Efstathopoulos and D.G. Katritsis, 2013. Reuse of devices in cardiology: Time for a reappraisal. Hellenic J. Cardiol., 54: 376-381.

Patterson, J.E., A.H. Sweeney, M. Simms, N. Carley and R. Mangi et al., 1995. An analysis of 110 serious enterococcal infections: Epidemiology, antibiotic susceptibility and outcome. Med. (Baltimore) 74: 191-200.

Penna, T.C.V. and C.A.M. Ferraz, 2001. Cleaning of blood-contaminated reprocessed angiographic catheters and spinal needles. Infection Control Hospital Epidemiol., 21: 499-504.

DOI: $10.1086 / 501793$
Pinto, T.J.A. and K.U. Graziano, 2000. Considerações Sobre Processamento De Artigos MédicoHospitalares De Uso Único. In: Infecção Hospitalar E Suas Interfaces Na Área Da Saúde, Fernandes, A.T. (Ed.), Editora Atheneu, São Paulo, ISBN-10: 8573792493.

Raad, I.I., M.F. Sabbagh, K.H. Rand and R.J. Sherertz, 1992. Quantitative tip culture methods and the diagnosis of central venous catheter-related infections. Diagnostic Microbiol. Infect. Dis., 15: 13-20. DOI: 10.1016/0732-8893(92)90052-U

Sandoe, J.A.T., I.R.H. Witherden, K.C. Au-Yeung, P. Kite and K.G. Kerr et al., 2002. Enterococcal intravascular catheter-related bloodstream infection: management and outcome of 61 consecutive cases. J. Antimicrobial Chemotherapy, 50: 577-582. DOI: $10.1093 / \mathrm{jac} / \mathrm{dkf1} 82$

Schinabeck, M.K. and M.A. Ghannoum, 2003. Catheterrelated infections-diagnosis, treatment and prevention. Clin. Microbiol. Newsletter, 25: 113118. DOI: $10.1016 / \mathrm{S} 0196-4399(03) 80036-3$

Shlaes, D.M., J. Levey and E. Wolinsky, 1981. Enterococcal bacteremia without endocarditis. Arch. Internal Med., 141: 578-581. DOI: $10.1001 /$ archinte. 1981.00340050030010

Shuman, E.K. and E.C. Chenoweth, 2012. Reuse of medical devices: Implications for infection control. Infect. Dis., Clinics North Am., 26: 165 172. PMID: 22284382

Silva, M.V. and T.J.A. Pinto, 2005. Reutilização simulada de produtos médico-hospitalares de uso único, submetidos à esterilização com óxido de etileno. Revista Brasileira de Ciências Farmacêuticas, 41: 181-190. DOI: $10.1590 /$ S1516-93322005000200005

Speziale, P., L. Visai, S. Rindi, G. Pietrocola and G. Provenza et al., 2008. Prevention and treatment of Staphylococcus biofilms. Curr. Med. Chem., 15: 3185-3195. PMID: 19075662 